\title{
Optimum Flapping Wing Motions of Dragonfly
}

\author{
By Yuichi Kamisawa and Koji IsOGAI \\ Micro Flying Robot Laboratory, Nippon Bunri University, Oita, Japan
}

(Received June 12th, 2007)

\begin{abstract}
We studied the optimum flapping wing motions of a dragonfly (Anax parthenope julius) from hovering to cruising flight at various speeds, using a 3D Navier-Stokes code coupled with an optimization algorithm. The minimum necessary power curve and optimum flapping wing motions for the various flight velocities were determined using the optimization algorithm. The minimum power curve shows the typical U-shape. The optimum flapping wing motions were evaluated by comparison with experimental data. Examining the flow patterns showed that the large-scale flow separation around the wings is suppressed at these optimum conditions, except for very low flight speeds including hovering.
\end{abstract}

Key Words: Dragonfly, CFD, Optimization, Flapping Wing

\section{Nomenclature}

$b_{\mathrm{F}}$ : semichord length at $75 \%$ semispan station of forewing

$b_{\theta}:$ rate of twist $\left(\theta=b_{\theta} z\right)$

$C_{\mathrm{df}}:$ drag coefficient of body

$D_{\mathrm{f}}$ : drag of body

$k$ : reduced frequency defined by $k=\left(b_{\mathrm{F}} \omega / V_{\mathrm{F}}\right)$

$L$ : lift acting in vertical (positive up) direction

$L_{X}$ : aerodynamic force acting in negative direction of $X$-axis

$L_{Y}$ : aerodynamic force acting along $Y$-axis (strokeplane)

$M$ : body mass

$n$ : load factor defined by $n=\bar{L} /(M g)$

$R e$ : Reynolds number defined by $R e=\left(b_{\mathrm{F}} V_{\mathrm{F}}\right) / v$

$X, Y, Z$ : Cartesian coordinates

$x, y, z:$ dimensionless Cartesian coordinates

$x_{\mathrm{p}}: x$-coordinate of axis of pitch

$T$ : thrust acting in horizontal direction

$T^{*}$ : excess thrust defined by $T^{*}=\bar{T} / D_{\mathrm{f}}$

$t$ : time

$t^{*}$ : dimensionless time defined by $t^{*}=t\left(V_{\mathrm{F}} / b_{\mathrm{F}}\right)$

$V_{\mathrm{F}}$ : reference velocity (maximum flapping velocity at $75 \%$ semispan station of forewing)

$V_{\infty}$ : free-stream velocity

$W$ : rate of work

$\lambda$ : phase advance angle of feathering motion ahead of flapping motion

$\varphi$ : flapping angle

$\varphi_{0}$ : flapping amplitude

$\varphi_{\mathrm{s}}$ : stroke-plane angle

$\theta$ : feathering angle

$\theta_{0}$ : feathering amplitude

$\nu$ : kinematical viscosity

$\xi, \eta, \zeta$ : dimensionless Cartesian coordinates in computational space $\psi$ : phase advance angle of flapping motion of hindwing ahead of forewing

Subscripts

F: forewing

$\mathrm{H}$ : hindwing

Superscripts

$\left({ }^{-}\right)$: time averaged (during one cycle) value

\section{Introduction}

It is well known that dragonflies have superior flying capabilities compared to other insects. For example, they can hover while maintaining their body axis horizontal, and can turn abruptly, etc. Many theoretical and experimental studies ${ }^{1,2)}$ have been conducted on the flapping-wing flight of dragonflies, but it is not well understood how they flap their wings in the various flight modes. Several parameters determine the flapping-wing motion of a dragonfly: the reduced frequency; stroke-plane angle; flapping amplitudes of the fore- and hindwings; feathering amplitudes of the fore- and hindwings; and phase angle between the flapping motions of the fore- and hindwings. These parameters change according to the specific flight mode. Azuma and Watanabe $^{3)}$ attempted to determine the necessary power curve of Anax parthenope julius using the LCM (Local Circulation Method) ${ }^{3)}$ coupled with experimentally determined aerodynamic data and wing motions. By modifying the experimentally observed feathering motions, they determined the necessary power curve for cruising flight at various flight velocities. However, the LCM they used is a potential theory, although the nonlinear behavior of the lift and moment due to viscous effects were taken partly into account by using the experimental data. Important unsteady viscous effects were not treated in the analysis and the wing motions used were not necessarily optimized.

It is of interest in studying dragonfly flight to determine theoretically the minimum necessary power curve and optimum wing motions corresponding to each flight mode, and 


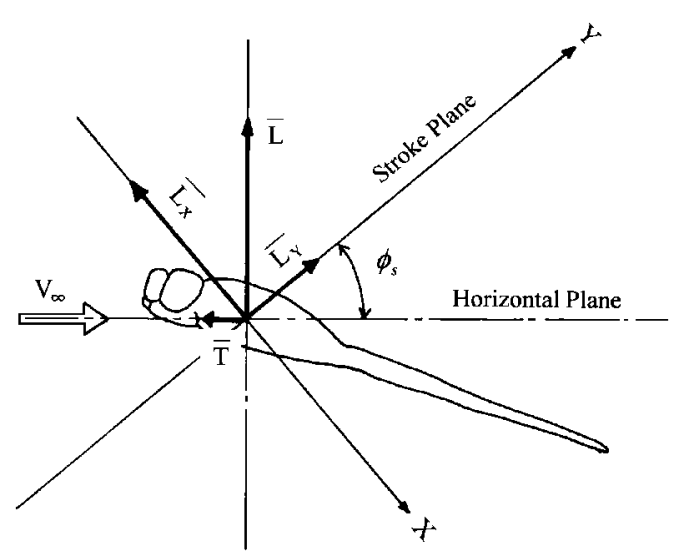

Fig. 1. Definitions of coordinates and aerodynamic forces.

then to determine whether such theoretical predictions agree with experimental observations. For this purpose, it is necessary to use the Navier-Stokes code to account for important unsteady viscous effects. This study investigates the minimum necessary power curve and the corresponding optimum flapping wing motions using the Navier-Stokes code coupled with an optimization algorithm.

\section{Analysis Method}

\subsection{Outline of Navier-Stokes code}

We used the 3D Navier-Stokes (N-S) code developed by Isogai, et al. ${ }^{4)}$ as an aerodynamic tool. The original N-S code, which was developed for computing the hovering state, has been modified to treat both hovering and cruising flight at any velocity.

Figure 1 shows the definitions of the coordinates and aerodynamic forces. The $Y$-axis is the stroke plane, along which each wing, namely, the fore- and hindwings, perform coupled flapping and feathering motion. The feathering motion advances $90^{\circ}$ ahead of the flapping motion. There is also a phase difference between the flapping motions of the foreand hindwings. The $Y$-axis is inclined at an angle (strokeplane angle $\varphi_{\mathrm{S}}$ ) to the horizontal line. The $X$-axis is taken as perpendicular to the $Y$-axis and the $Z$-axis is taken as the spanwise direction. (Hence, a left-handed Cartesian coordinate system is used.) $V_{\infty}$ is the free-stream velocity along the horizontal direction. $\bar{L}_{Y}$ is the time-mean aerodynamic force acting along the $Y$-axis and $\bar{L}_{X}$ is the time-mean aerodynamic force in the negative direction along the $X$-axis. From these definitions of the forces, the time-mean lift $\bar{L}$ acting in the vertical direction and the time-mean thrust $\bar{T}$ acting in the horizontal direction are given by

$$
\begin{aligned}
& \bar{L}=\bar{L}_{X} \cos \varphi_{\mathrm{S}}+\bar{L}_{Y} \sin \varphi_{\mathrm{S}} \\
& \bar{T}=\bar{L}_{X} \sin \varphi_{\mathrm{S}}-\bar{L}_{Y} \cos \varphi_{\mathrm{S}}
\end{aligned}
$$

To compute cruising flight, $\bar{L}_{X}$ and $\bar{L}_{Y}$ are computed using the N-S code for the given $V_{\infty}$ and $\varphi_{\mathrm{S}}$, and then $\bar{L}$ and $\bar{T}$ are computed by Eqs. (1) and (2). Note that $V_{\infty}$ and $\bar{T}$ should be 0 for hovering flight. Hence, $\varphi_{\mathrm{S}}$ can be determined from Eq. (2) as $\varphi_{\mathrm{S}}=\tan ^{-1}\left(\bar{L}_{Y} / \bar{L}_{X}\right)$. The similarity parameters governing the unsteady viscous flow around the present

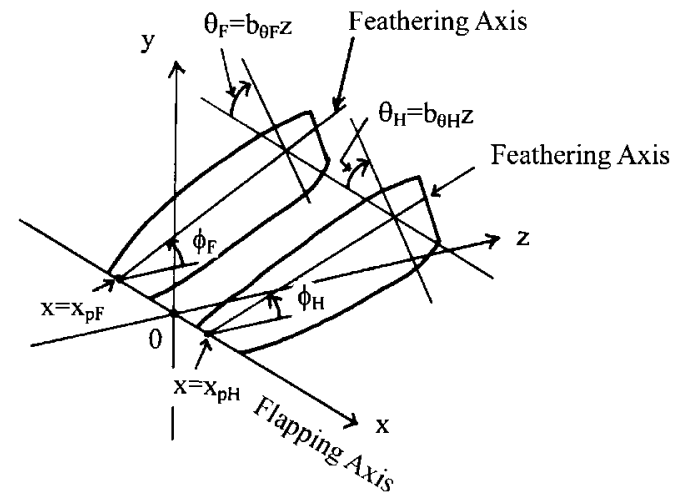

Fig. 2. Definitions of wing motions of fore- and hindwings.

tandem wing configuration are the Reynolds number $R e$, and the reduced frequency $k$, defined by:

$$
\begin{aligned}
& R e=\left(b_{\mathrm{F}} V_{\mathrm{F}}\right) / v \\
& k=\left(b_{\mathrm{F}} \omega\right) / V_{\mathrm{F}}
\end{aligned}
$$

The motions of the fore- and hindwings, which are coupled flapping and feathering motions, can be expressed as displacements of the wing surfaces in the $y$ direction as follows. (See also Fig. 2 for the definitions of wing motions.)

Forewing:

$$
\begin{aligned}
y= & \left(\varphi_{0, \mathrm{~F}} z\right) \sin k t^{*} \\
& -\left(b_{\theta, \mathrm{F}} z\right)\left(x-x_{\mathrm{p}, \mathrm{F}}\right) \sin \left(k t^{*}+\lambda_{\mathrm{F}}\right)
\end{aligned}
$$

Hindwing:

$$
\begin{aligned}
y= & \left(\varphi_{0, \mathrm{H}} z\right) \sin \left(k t^{*}+\psi\right) \\
& -\left(b_{\theta, \mathrm{H}} z\right)\left(x-x_{\mathrm{p}, \mathrm{H}}\right) \sin \left(k t^{*}+\lambda_{\mathrm{H}}+\psi\right)
\end{aligned}
$$

All the physical quantities in the N-S equations are nondimensionalized by the semichord length $b_{\mathrm{F}}$, the maximum heaving velocity $V_{\mathrm{F}}$ (which is caused by the flapping oscillation) at the $75 \%$ semispan station of the forewing, and the air density.

To handle the present two-wing problem in which each wing moves differently, a multi-block method is used. The physical space is divided into four sectors, denoted as I, II, III, and IV, as shown in Fig. 3(a). Each sector in the physical space moves and deforms with time in accordance with the wing motion and can be mapped to a corresponding sector in the computational spaces shown in Fig. 3(b). The time-differenced form of the geometric conservation law ${ }^{5)}$ coupled with the conservation form of the $3 \mathrm{D} \mathrm{N}-\mathrm{S}$ equations in each computational space is solved at each time step. The far-field boundaries are located 15 chord lengths away from the wing in the $x$ and $y$ directions and five-semispan lengths away in the $z$ direction. At these boundaries the flow quantities are given by the zero-order extrapolation from the inner points for hovering and the dimensionless velocities $\left(V_{\infty} / V_{\mathrm{f}}\right) \sin \varphi_{\mathrm{s}}$ and $\left(V_{\infty} / V_{\mathrm{f}}\right) \cos \varphi_{\mathrm{s}}$ for cruising flight with finite advance velocities. Since the Reynolds number of the present dragonfly problem is on the order of $10^{3}$, the turbulence model is not used in the present computations. Further details of the method are explained elsewhere. ${ }^{4)}$ 

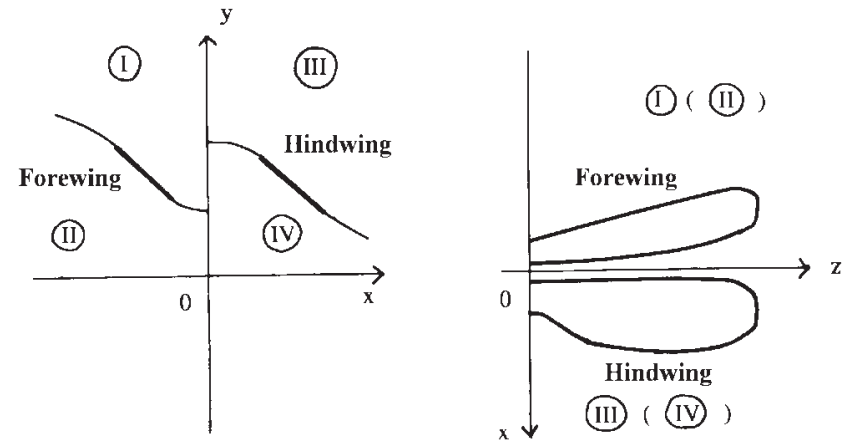

(a)
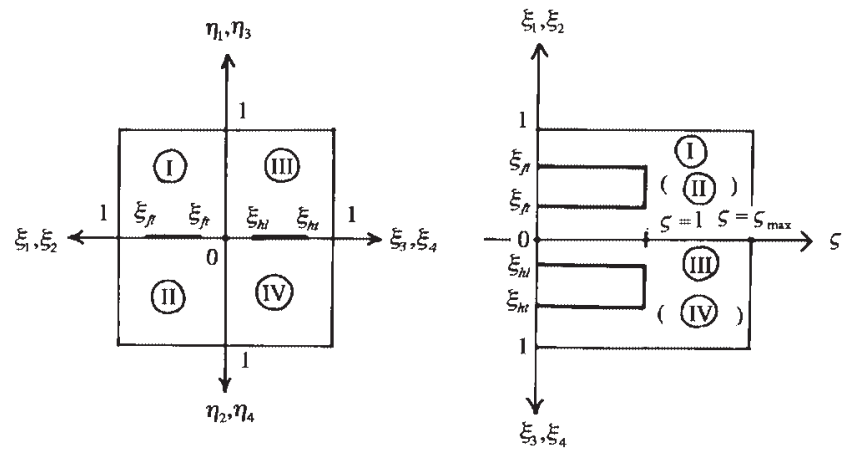

(b)

Fig. 3. (a) Physical spaces. (b) Computational spaces.

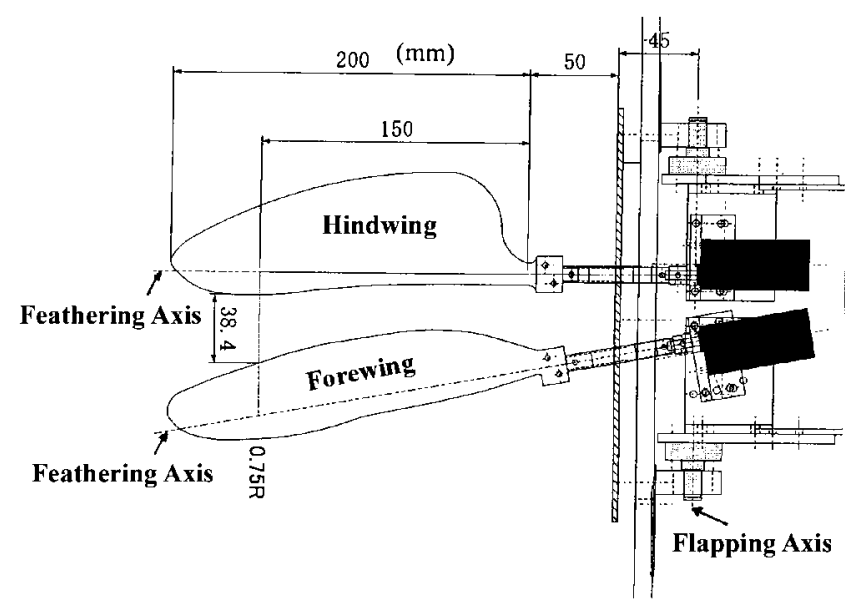

Fig. 4. Planform and arrangement of flapping tandem wing configuration. ${ }^{6)}$

Although the reliability and accuracy of the present N-S code were extensively examined by comparison with experimental data, ${ }^{4,6)}$ here we cite some other results ${ }^{6)}$ to demonstrate the fidelity of the present N-S code. Yamamoto et al. ${ }^{6}$ measured the unsteady fluid dynamic forces acting on the mechanical dragonfly model at the hovering state. They compared the experimental data with the computation obtained using the present N-S code. Figure 4 shows the planforms and arrangement of the experimental wings. The fore- and hindwings, whose planforms are similar to those of A. p. julius are oscillated in coupled flapping and feathering motion in the water tank. The similarity parameters $(R e$ and $k)$ are approximately matched with those of the

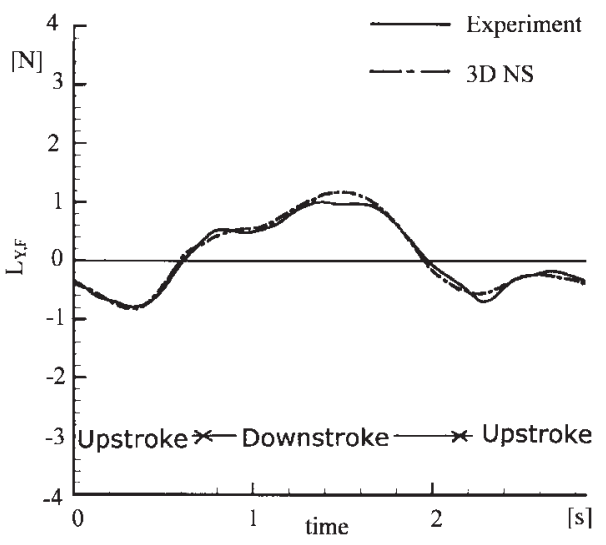

(a)

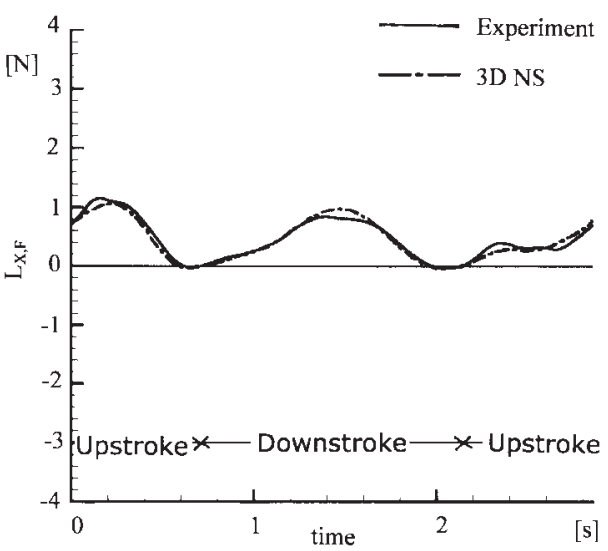

(b)

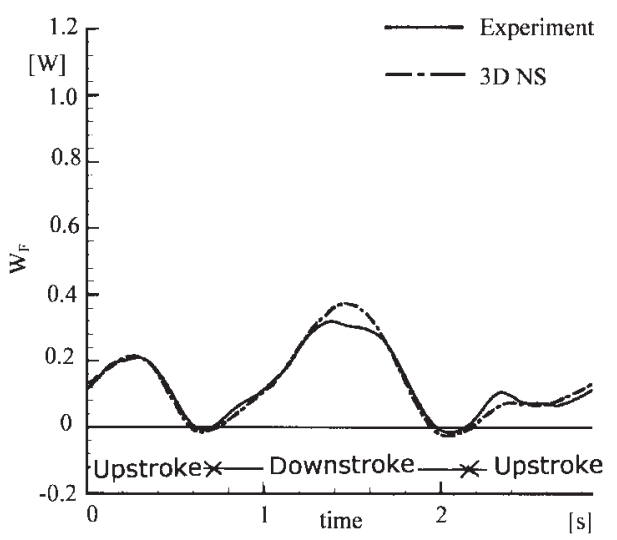

(c)

Fig. 5. $L_{Y, \mathrm{~F}}, L_{X, \mathrm{~F}}$, and $W_{\mathrm{F}}$ during one cycle of oscillation for $\psi=40 \mathrm{deg}$ for forewing. ${ }^{6)}$

real dragonfly. The values of $R e$ and $k$ in this experiment are $5.63 \times 10^{3}$ and $k=0.135$, respectively. For further details of this experimental work are presented elsewhere. ${ }^{6}$ )

Figures 5 and 6 compare the time histories of $L_{Y, \mathrm{~F}}, L_{X, \mathrm{~F}}$ and $W_{\mathrm{F}}$ for the forewing, and $L_{Y, \mathrm{H}}, L_{X, \mathrm{H}}$ and $W_{\mathrm{H}}$ for the hindwing with those values obtained by the same N-S code as used in this work. As seen in the figures, the experimental data and N-S computations show excellent agreement. In the work by Yamamoto et al., ${ }^{6}$ the time averaged values of $L_{X}$, $L_{Y}$ and $W$ also show good agreement between the experiment and computation. 


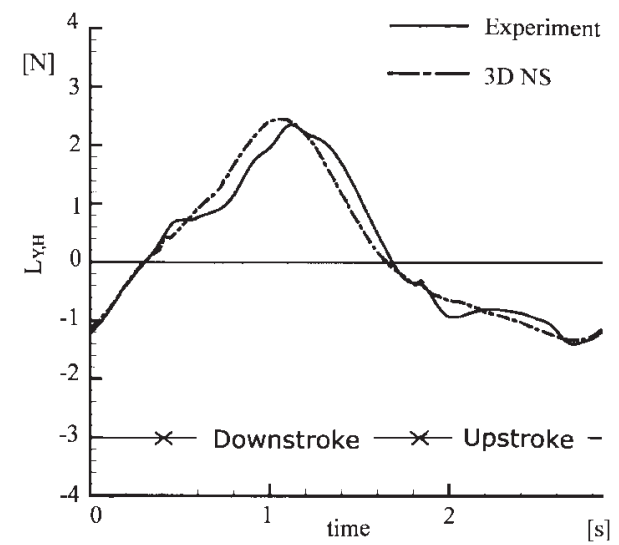

(a)

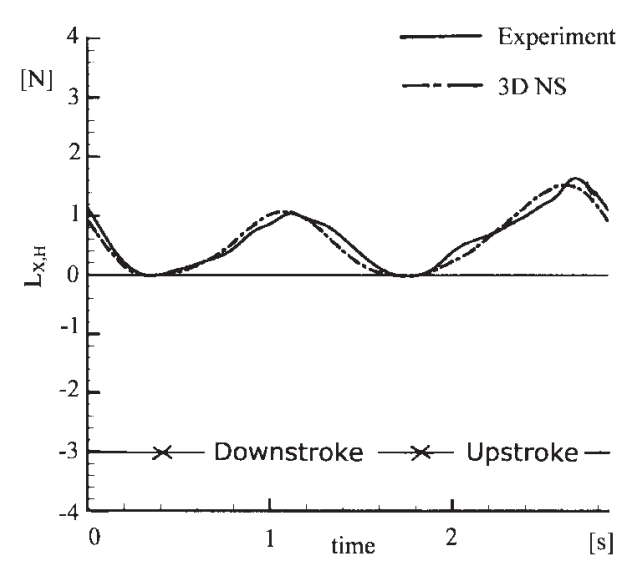

(b)

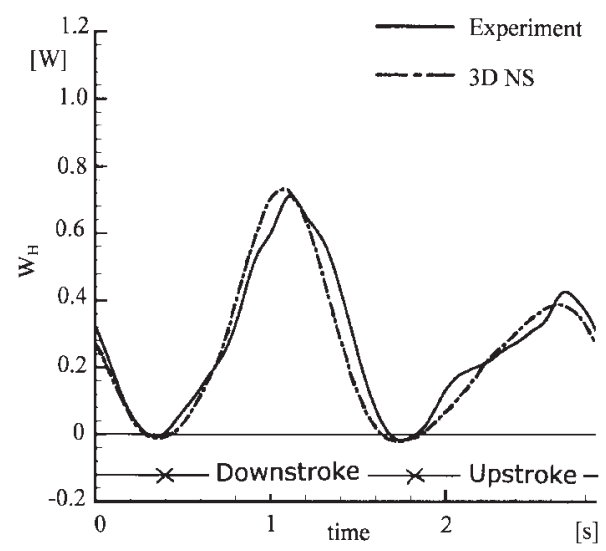

(c)

Fig. 6. $L_{X, \mathrm{H}}, L_{Y, \mathrm{H}}$ and $W_{\mathrm{H}}$ during one cycle of oscillation for $\psi=40^{\circ}$ for hindwing. ${ }^{6)}$

\subsection{Dragonfly model and optimization procedure}

We used A. $p$. julius as a typical dragonfly model for the optimum flapping wing motion study. According to Azuma and Watanabe, ${ }^{3)}$ the full span length of the forewing is about $0.10 \mathrm{~m}$ and the aspect ratio is 10 . The planforms of the foreand hindwings used in the present computations are shown in Fig. 7. The semichord length at the $75 \%$ semispan station of the forewing is $0.005 \mathrm{~m}$, which is used as the reference length $b_{\mathrm{f}}$. Table 1 shows the basic characteristics of $A$. p. julius. ${ }^{3)}$

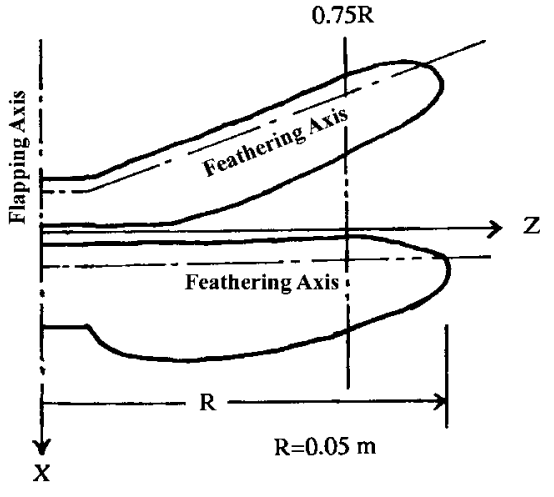

Fig. 7. Planform of dragonfly model.

Table 1. Basic characteristics of Anax parthenope julius. ${ }^{3)}$

\begin{tabular}{lcc}
\multicolumn{1}{c}{ Characteristics } & Unit & Value \\
\hline $\begin{array}{l}\text { Mass } \\
\text { Semichord length } b_{\mathrm{f}}\end{array}$ & {$[\mathrm{kg}]$} & $7.9 \times 10^{-4}$ \\
$\quad$ at 75\% semispan station of forewing & {$[\mathrm{m}]$} & 0.005 \\
$\begin{array}{l}\text { Fullspan } \\
\text { Flapping frequency }\end{array}$ & {$[\mathrm{m}]$} & 0.10 \\
\hline
\end{tabular}

Table 2. Design parameters.

\begin{tabular}{cl}
\hline$\varphi_{\mathrm{S}}$ & Stroke-plane angle \\
$\varphi_{0 \mathrm{~F}}$ & Flapping amplitude of forewing \\
$\varphi_{\mathrm{OH}}$ & Flapping amplitude of hindwing \\
$b_{\theta \mathrm{FU}}$ & Rate of twist of forewing in upward motion \\
$b_{\theta \mathrm{FD}}$ & Rate of twist of forewing in downward motion \\
$b_{\theta \mathrm{HU}}$ & Rate of twist of hindwing in upward motion \\
$b_{\theta \mathrm{HD}}$ & Rate of twist of hindwing in downward motion \\
$\psi$ & Phase advance angle of flapping motion of hindwing \\
\hline
\end{tabular}

As seen in Eqs. (5) and (6), several parameters define the wing motion of the dragonfly. According to Azuma and Watanabe, ${ }^{3)}$ the flapping frequency is held constant at $28 \mathrm{~Hz}$ independent of flight velocity, and the phase advance angle of the feathering motion ahead of the flapping motion of each wing $\left(\lambda_{\mathrm{F}}\right.$ and $\left.\lambda_{\mathrm{H}}\right)$ is also constant at about $90^{\circ}$.

All the other parameters change, depending on the flight velocities. In this study, we select the eight parameters in Table 2 as the design parameters.

The objective function of the present problem is the timemean necessary power. It should be noted that the timemean lift and thrust generated by the wing motion should sustain its own weight and cope with drag of the body, respectively. (Although note that the presence of the body is not accounted for in the present N-S computation.) Consequently, the present optimization problem is to find or determine the eight parameters that define the optimum wing motion and that require the minimum necessary power while satisfying the constraints imposed on lift and thrust.

We use a complex method proposed originally by Box ${ }^{7)}$ as the optimization algorithm. It is a direct-search method that can handle multiple constraints without using gradients. 


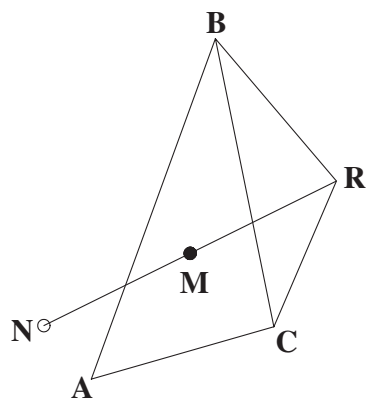

A, B, C, R : Vertices

$R$ : Rejected vertex

Fig. 8. Complex method.

Table 3. Constraints.

$\begin{array}{ll}\bar{L} \geqslant M g & \text { Time mean lift should be greater than own weight } \\ \bar{T} \geqslant D_{\mathrm{f}} & \text { Time mean thrust should be greater than body drag }\end{array}$

In this method, the optimization proceeds by using flexible figures of $K \geqslant N+1$ vertices in $N$-dimensional design spaces, where $N$ is the number of design variables. In Fig. 8, four vertices (points A, B, C, and R) are shown for illustration.

Each vertex in the complex model corresponds to a specific wing motion specified by the eight design variables in the present flapping wing problem. The objective function (time-mean necessary power) and constraint functions (lift and thrust) at each vertex are evaluated using the N-S code. The worst vertex (vertex giving the highest necessary power among the four vertices, indicated by point $\mathrm{R}$ in Fig. 8) is then found and rejected, and a new point $\mathrm{N}$ is located at a distance $\alpha d$ from the centroid $\mathrm{M}$, in the opposite direction to $\mathrm{R}$, of the vertices $\mathrm{A}, \mathrm{B}$, and $\mathrm{C}$, where $d$ is the distance between $\mathrm{M}$ and $\mathrm{R}$, and $\alpha$ is a coefficient used to accelerate the convergence, usually taken to be $\alpha=1.0-1.3$. This process is called Rule 1 . The objective functions and constraint functions at the new point $\mathrm{N}$ are then evaluated with the N-S code. If any of the constraint functions do not satisfy the specified value of the constraints, $\mathrm{N}$ is shifted toward $\mathrm{M}$ to a distance half that of the original distance. This process is Rule 2 and is repeated until all the constraints are satisfied. Rule 1 and Rule 2 are repeated until the objective function converges. The constraints imposed in the present problem are shown in Table 3.

In Table $3, D_{\mathrm{f}}$ represents the drag of the body. Note that drag acting on the wing is already subtracted in computing the time-mean thrust $\bar{T}$. $D_{\mathrm{f}}$ is evaluated using the following formula proposed by Azuma and Watanabe: ${ }^{3)}$

$$
D_{\mathrm{f}}=\frac{1}{2} \rho V_{\infty}^{2} S_{\mathrm{f}} C_{\mathrm{Df}}
$$

where, $S_{\mathrm{f}}$ is the maximum section area of the body and $C_{\text {Df }}=1.25$, obtained by a wind-tunnel experiment. ${ }^{3)}$

The details of the optimization algorithm are presented as a flowchart in Fig. 9. As shown, we set the first vertex initially. The first vertex for which $\bar{L}$ and $\bar{T}$ are evaluated using the N-S code should satisfy the constraints. To set the first vertex, we referred to the wing motions measured by Azuma

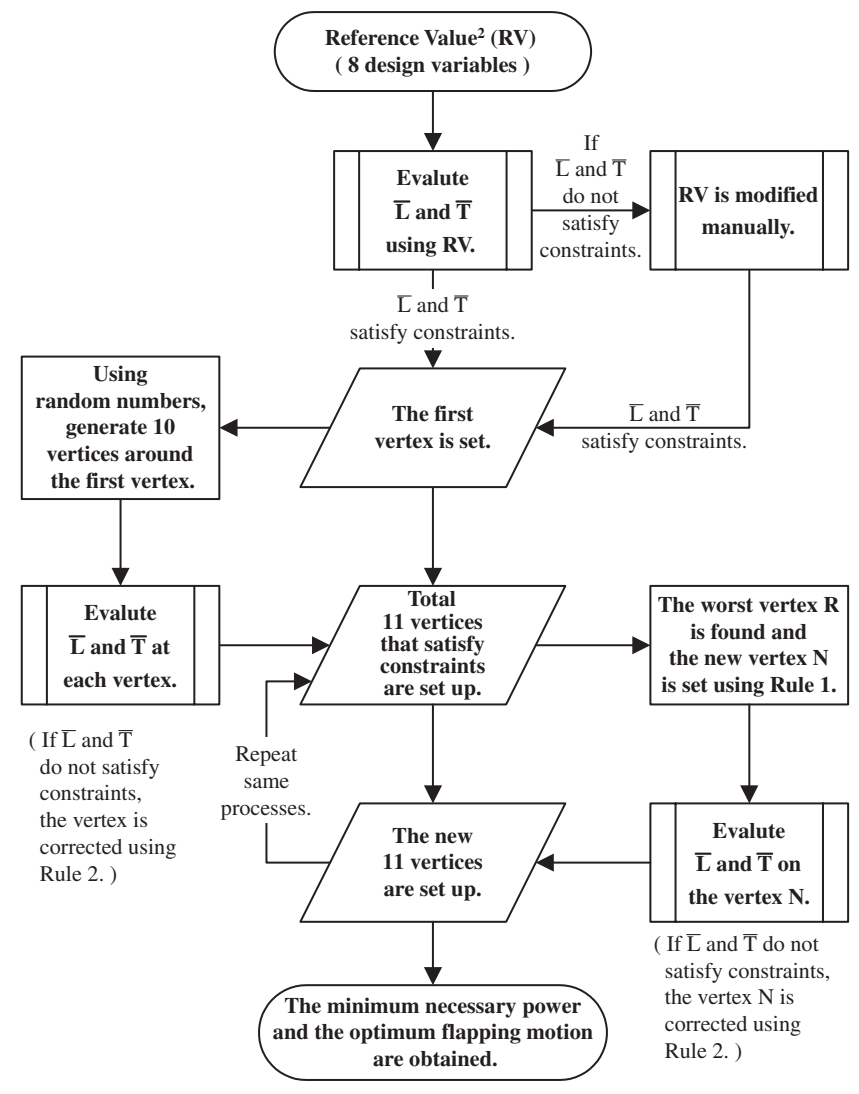

Fig. 9. Flowchart of optimization procedure.

Table 4. Results of N-S simulations for wing motions measured by windtunnel experiment. ${ }^{3)}$

\begin{tabular}{lcccc}
\hline \multicolumn{1}{c}{$V_{\infty}$} & $0.7 \mathrm{~m} / \mathrm{s}$ & $1.5 \mathrm{~m} / \mathrm{s}$ & $2.3 \mathrm{~m} / \mathrm{s}$ & $3.2 \mathrm{~m} / \mathrm{s}$ \\
\hline $\bar{L}(\mathrm{~N})$ & $5.18 \times 10^{-3}$ & $7.6 \times 10^{-3}$ & $1.25 \times 10^{-2}$ & $1.79 \times 10^{-2}$ \\
$n$ & 0.669 & 0.981 & 1.613 & 2.312 \\
$\bar{T}(\mathrm{~N})$ & $4.39 \times 10^{-4}$ & $-2.62 \times 10^{-3}$ & $-5.49 \times 10^{-3}$ & $-3.08 \times 10^{-3}$ \\
$D_{\mathrm{f}}(\mathrm{N})$ & $2.81 \times 10^{-5}$ & $1.29 \times 10^{-4}$ & $3.04 \times 10^{-4}$ & $5.88 \times 10^{-4}$ \\
$T^{*}$ & 15.617 & -20.310 & -18.090 & -5.230 \\
$W(\mathrm{~W})$ & 0.018 & 0.011 & 0.014 & 0.025 \\
\hline
\end{tabular}

and Watanabe ${ }^{3)}$ in wind-tunnel experiments. However, using the N-S computations we found that the wing motions measured by Azuma and Watanabe ${ }^{3)}$ do not satisfy the constraints on lift and thrust. Table 4 shows the time-mean aerodynamic data computed by the N-S code for the wing motions measured at various flight velocities.

As seen in the table, none of the values satisfy the constraints, that is, the load factor is less than 1.0 for $V_{\infty}=0.7 \mathrm{~m} / \mathrm{s}$ and $1.5 \mathrm{~m} / \mathrm{s}$, and the excess thrust $\bar{T}^{*}$ is negative for $V_{\infty}=1.5 \mathrm{~m} / \mathrm{s}, 2.3 \mathrm{~m} / \mathrm{s}$, and $3.2 \mathrm{~m} / \mathrm{s}$. Therefore, the wing motions measured by Azuma and Watanabe ${ }^{3)}$ must be corrected. Such corrections were made by trial and error until all the constraints were satisfied. Table 5 shows the measured and corrected values of the eight design variables that define the wing motion at $V_{\infty}=0.7 \mathrm{~m} / \mathrm{s}$.

The manual corrections gave a load factor and excess thrust of 1.198 and 21.570. Making similar corrections, we set the first vertex for the other velocities. The subsequent 
Table 5. Measured and corrected design variables.

\begin{tabular}{cccc}
\hline $\begin{array}{c}\text { Design } \\
\text { variable }\end{array}$ & $\begin{array}{c}\text { Reference } \\
\text { value }^{3)}\end{array}$ & $\begin{array}{c}\text { Initial } \\
\text { value }\end{array}$ & $\begin{array}{c}\text { Amount of } \\
\text { correction }\end{array}$ \\
\hline$\varphi_{\mathrm{S}}$ & $39^{\circ}$ & $31^{\circ}$ & $-8^{\circ}$ \\
$\varphi_{0 \mathrm{~F}}$ & $36^{\circ}$ & $51^{\circ}$ & $+15^{\circ}$ \\
$\varphi_{0 \mathrm{H}}$ & $26^{\circ}$ & $41^{\circ}$ & $+15^{\circ}$ \\
$\theta_{\mathrm{OFU}}$ & $54^{\circ}$ & $54^{\circ}$ & $0^{\circ}$ \\
$\theta_{0 \mathrm{FD}}$ & $54^{\circ}$ & $54^{\circ}$ & $0^{\circ}$ \\
$\theta_{0 \mathrm{HU}}$ & $38^{\circ}$ & $38^{\circ}$ & $0^{\circ}$ \\
$\theta_{0 \mathrm{HD}}$ & $38^{\circ}$ & $38^{\circ}$ & $0^{\circ}$ \\
$\psi$ & $51^{\circ}$ & $41^{\circ}$ & $-10^{\circ}$ \\
\hline
\end{tabular}

vertices were generated around the first vertex using random numbers. Rule 2 is used to correct the location of the vertices if the constraints at any of the vertices are violated. We then get the first set of vertices (11 vertices are used in the present problem) and proceed with the optimization process using Rule 1 and Rule 2 until the converged value of the objective function is obtained. (See further details of the procedure in Fig. 8.) The convergence is identified when the decrement of the necessary power becomes less than $0.001 \mathrm{~W}$ for 11 iteration cycles.

Figures 10(a)-(c) show the convergence histories of the necessary power $\bar{W}$, load factor, and excess thrust $\bar{T}^{*}$, respectively, for each of the 11 vertices for $V_{\infty}=4 \mathrm{~m} / \mathrm{s}$.

\section{Results}

\subsection{Minimum necessary power curve}

Figure 11 shows the minimum necessary power curve for $V_{\infty}=0-8 \mathrm{~m} / \mathrm{s}$, obtained by applying the optimization procedure described in the previous section. In the figure, the necessary powers obtained by the wind-tunnel experiment of Azuma and Watanabe ${ }^{3)}$ are also shown for $V_{\infty}=$ $0.7 \mathrm{~m} / \mathrm{s}, 1.5 \mathrm{~m} / \mathrm{s}, 2.3 \mathrm{~m} / \mathrm{s}$ and $3.2 \mathrm{~m} / \mathrm{s}$. The necessary power curve $^{3)}$ computed using the LCM and experimentally obtained data for flapping motions is also shown by the dotted line for comparison. The available power of $A$. $p$. julius is estimated to be $5.14 \times 10^{-2} \mathrm{~W}$ by the following formulas: ${ }^{3)}$

$$
P_{\mathrm{A}} / m_{\mathrm{m}}=260 \mathrm{~W} / \mathrm{kg}, \quad m_{\mathrm{m}} / m=1 / 4
$$

where, $m_{\mathrm{m}}$ is the muscle mass of the dragonfly and $m$ is the total mass. Hence, the minimum necessary power obtained by the present optimization is less than the available power for $V_{\infty}=0-8 \mathrm{~m} / \mathrm{s}$. The necessary power for hovering is $3.8 \times 10^{-2} \mathrm{~W}$ and the lowest necessary power is $1.1 \times$ $10^{-2} \mathrm{~W}$ at $V_{\infty}=4 \mathrm{~m} / \mathrm{s}$, which is about $1 / 3$ that for hovering. The necessary power for $V_{\infty}=8 \mathrm{~m} / \mathrm{s}$ is $4.6 \times$ $10^{-2} \mathrm{~W}$, which is about 1.2 times that for hovering.

As seen in the figure, the necessary powers obtained by the present computation show good agreement with those of the wind-tunnel experiment and the LCM results for $V_{\infty}=0-2.0 \mathrm{~m} / \mathrm{s}$. However, the mismatch increases with increasing flight velocity. That is, the flight velocity at the minimum power is $V_{\infty}=1.5 \mathrm{~m} / \mathrm{s}$ for the LCM prediction, but it is $V_{\infty}=4 \mathrm{~m} / \mathrm{s}$ for the present computation, and the

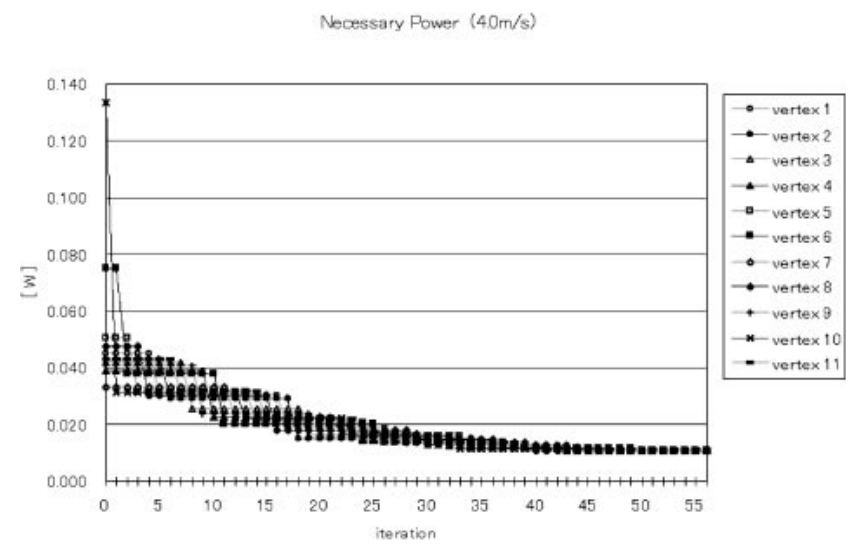

(a) Necessary power

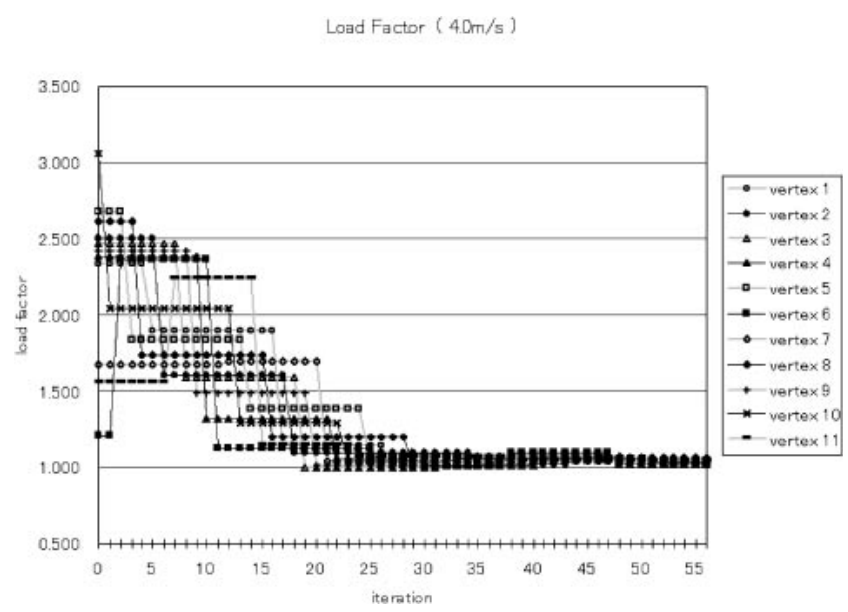

(b) Load factor

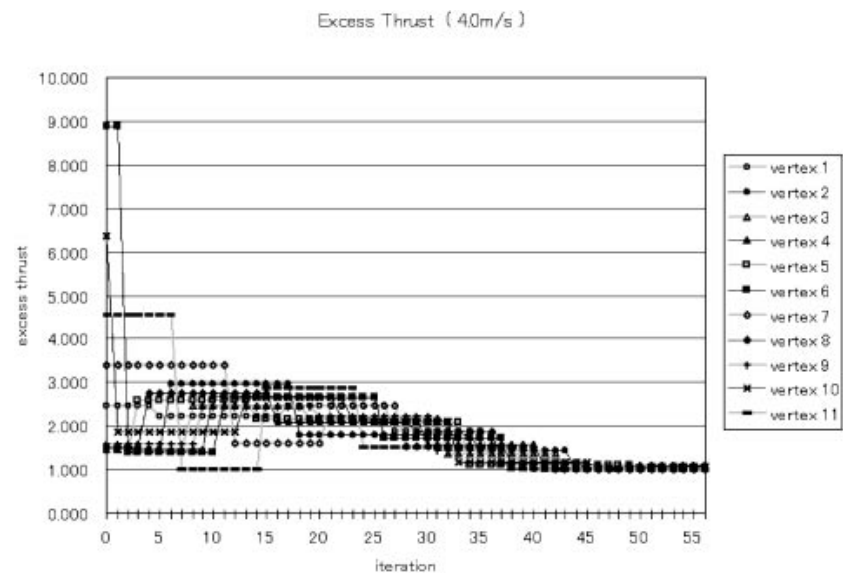

(c) Excess thrust

Fig. 10. Convergence histories of objective function and constraint functions for $V_{\infty}=4.0 \mathrm{~m} / \mathrm{s}$.

maximum velocity estimated by the present computation is about $1.5 \mathrm{~m} / \mathrm{s}$ higher than that estimated by Azuma and Watanabe. ${ }^{3)}$ This discrepancy might be due to the fact that the LCM used by Azuma and Watanabe ${ }^{3)}$ was a potential theory (although the non-linear behavior of lift and moment due to viscous effects were partly taken into account by using experimental data) and the important unsteady viscous effects were not treated in their analyses and the wing motions used were not necessarily optimum. 


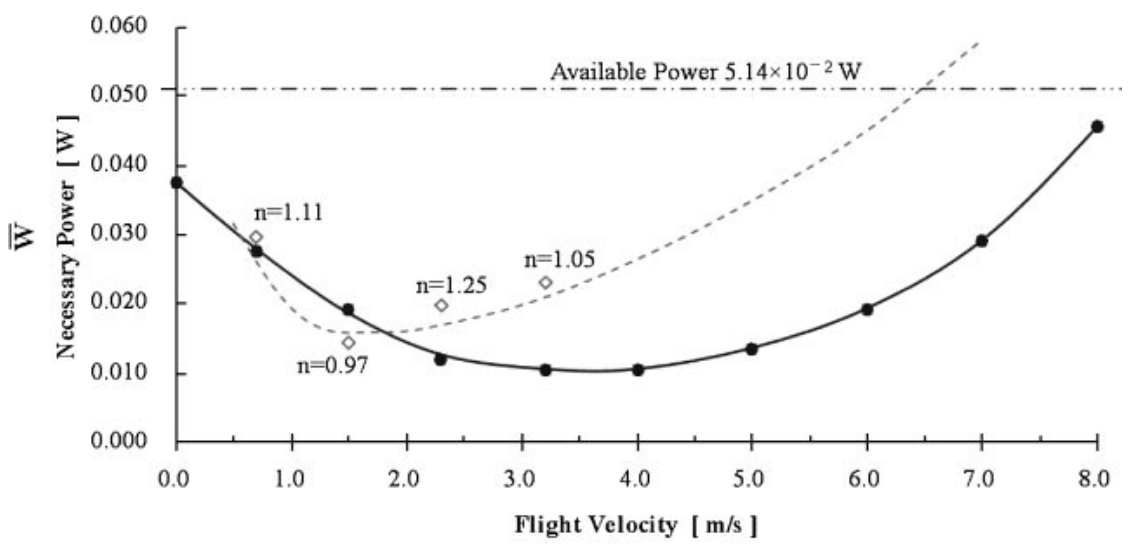

- Present $(\mathrm{n} \fallingdotseq 1) \diamond$ Experiment (Azuma \& Watanabe ${ }^{2}$ ) - - - ; LCM (Azuma \& Watanabe ${ }^{2}$ )

Fig. 11. Comparison of minimum necessary power curves.

\subsection{Optimum flapping wing motions}

In Figs. 12(a)-(h), the behaviors of the eight design variables specifying the optimum wing motions, namely, $\varphi_{\mathrm{S}}$, $\varphi_{0 \mathrm{~F}}, \varphi_{0 \mathrm{H}}, b_{\theta \mathrm{FU}}, b_{\theta \mathrm{FD}}, b_{\theta \mathrm{HU}}, b_{\theta \mathrm{HD}}$, and $\psi$, are plotted with respect to flight velocity. The solid lines show the converged values of the present computations. The figures also show the experimental results of Azuma and Watanabe ${ }^{3)}$ for $V_{\infty}=0.7 \mathrm{~m} / \mathrm{s}, \quad V_{\infty}=1.5 \mathrm{~m} / \mathrm{s}, \quad V_{\infty}=2.3 \mathrm{~m} / \mathrm{s}$ and $V_{\infty}=$ $3.2 \mathrm{~m} / \mathrm{s}$ for comparison. These experimental results were obtained for free-flight of A. p. julius in the wind-tunnel. They analyzed the data on wing motions taken by highspeed camera.

As seen in Fig. 12(a), the stroke-plane angle $\varphi_{\mathrm{S}}$ is $7.7^{\circ}$ for hovering and increases rapidly with increasing flight velocity, becoming $87.7^{\circ}$ at $V_{\infty}=8.0 \mathrm{~m} / \mathrm{s}$. The agreement between the present results with the wind-tunnel experiment is good for $V_{\infty}=0.7 \mathrm{~m} / \mathrm{s}$ and $1.5 \mathrm{~m} / \mathrm{s}$, but not so good for $2.3 \mathrm{~m} / \mathrm{s}$ and $3.2 \mathrm{~m} / \mathrm{s}$. The flapping amplitudes, shown in Figs. 12(b) and (c), of fore- and hindwings, namely, $\varphi_{0 \mathrm{~F}}$ and $\varphi_{0 \mathrm{H}}$, show the similar behavior to those of the experiment but there are some quantitative discrepancies. The behaviors of the rate of twist, $b_{\theta \mathrm{F}}$ and $b_{\theta \mathrm{H}}$, shown in Figs. 12(d)-12(g), show the qualitative agreement with the experiment except at $V_{\infty}=0.7 \mathrm{~m} / \mathrm{s}$, where large quantitative discrepancies are observed. As a whole, the rate of twist decreases with increasing flight velocity. For phase angle between the flapping motions of the fore- and hindwings shown in Fig. 12(h), the agreement with the experimental data is good except at $V_{\infty}=0.7 \mathrm{~m} / \mathrm{s}$. The phase angle takes values of $30^{\circ}-40^{\circ}$ for low flight velocities and high values of $80^{\circ}-90^{\circ}$ for $V_{\infty}=3.2-8.0 \mathrm{~m} / \mathrm{s}$.

For optimum flapping wing motions predicted by the present optimization study, the agreement with real dragonfly motions in free-flight conditions, observed in the windtunnel experiment is only qualitative. However, it should be noted that it is difficult to obtain rigorous data for a real dragonfly for the cruising condition of $n=1$ in free flight. The data obtained by Azuma and Watanabe ${ }^{3)}$ are not obtained at exactly the $n=1$ condition.

\section{Flow Patterns and Time Histories of Lift and Thrust}

Figures 13 show the flow patterns (iso-vorticity distributions) around the fore- and hind-right-wings at $V_{\infty}=0 \mathrm{~m} / \mathrm{s}$. The stroke-plane angle $\varphi_{\mathrm{S}}=7.7^{\circ}$ for this case. These figures show the flow patterns when the flapping velocity of the forewing becomes maximum. Flow separation from the leading edges of both the fore- and hindwings is observed. Figure 14 shows the time histories of lift and thrust during one oscillation cycle for $V_{\infty}=0 \mathrm{~m} / \mathrm{s}$.

It should be noted that the lift is the vector sum of $L_{X}$ and $L_{Y}$ (Eqs. (1) and (2)). Therefore most of the lift $(L)$ acting in the vertical direction is generated by the contribution of $L_{X}$ and the thrust $(T)$ acting in the horizontal direction is generated by the contribution of $L_{Y}$ for this hovering condition. The time averaged values of $L, T$ and $W$ are $0.00763 \mathrm{~N}$ $(n=0.99), 0.00173 \mathrm{~N}$ and $0.0381 \mathrm{~W}$, respectively.

Figures 15 show the flow patterns around the fore- and hind-right-wings at $V_{\infty}=4 \mathrm{~m} / \mathrm{s}$. Large-scale flow separations are suppressed, perhaps because the effective angle of attack for both the fore- and hindwings becomes sufficiently small to prevent flow separation for optimum flapping wing motions. This seems to be the main reason why the minimum power curve shows the minimum at this optimum condition because the large-scale flow separation consumes large amounts of power. Figure 16 shows the time histories of lift and thrust at $V_{\infty}=4.0 \mathrm{~m} / \mathrm{s}$.

The time-averaged values of lift, thrust and necessary power for this case are $8.28 \times 10^{-3} \mathrm{~N}(n=1.07), 9.4 \times$ $10^{-4} \mathrm{~N}\left(T^{*}=1.02\right)$ and $0.0108 \mathrm{~W}$, respectively.

Figures 17 show the flow patterns around the fore- and hind-right-wings at $V_{\infty}=8.0 \mathrm{~m} / \mathrm{s}$.

As shown in the figures, the large-scale flow separation is also suppressed in this condition.

Figure 18 shows the time histories of lift and thrust at $V_{\infty}=8.0 \mathrm{~m} / \mathrm{s}$. There are some wavy patterns in the variations of lift and thrust. Why is still unknown but perhaps some sort of wake instability, such as Karman vortex, might cause this phenomenon. The time averaged values of $L, T$ 

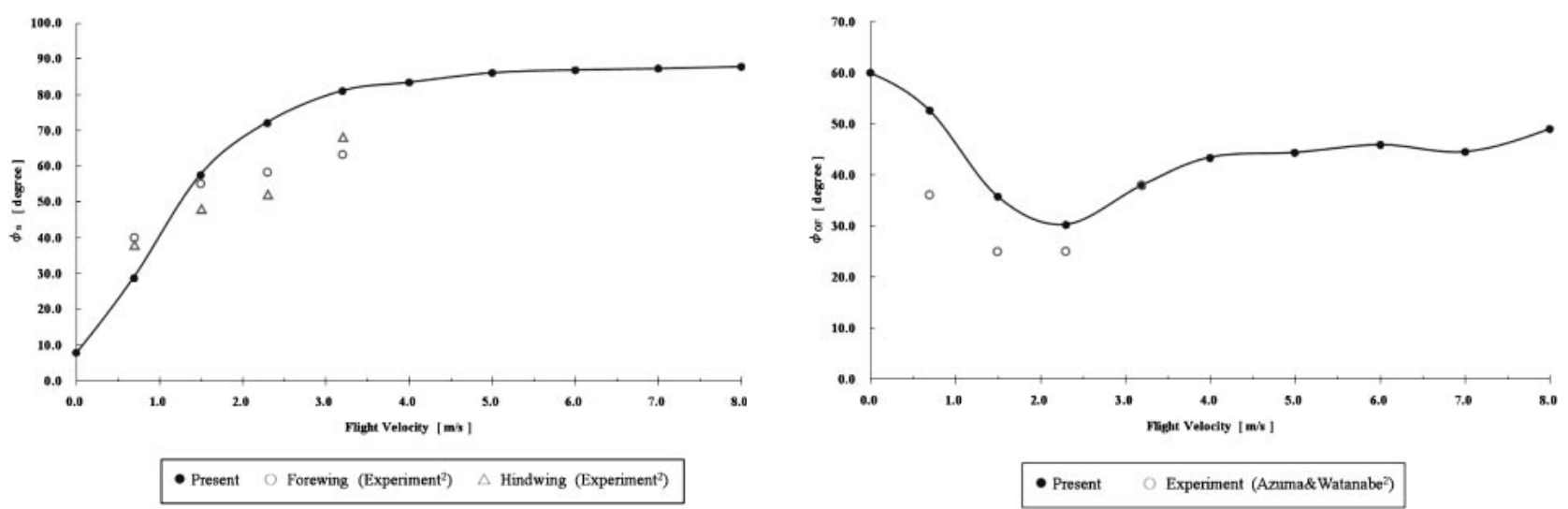

(a)

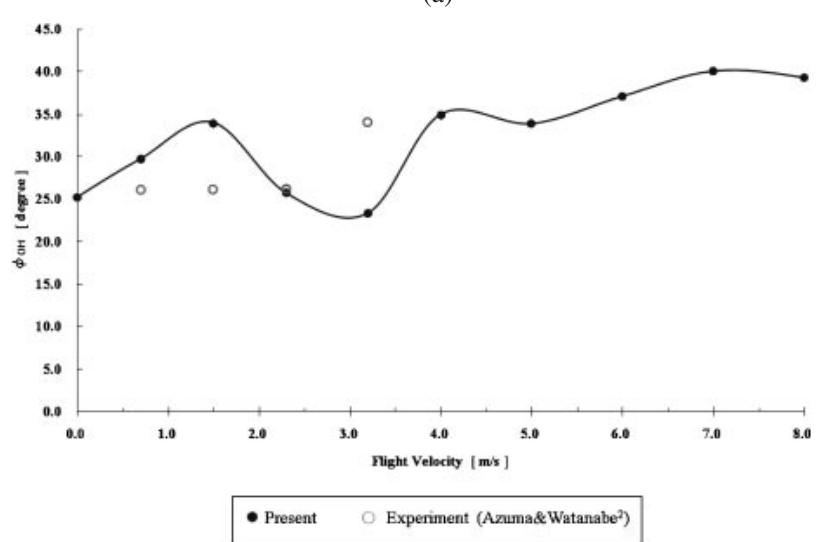

(b)

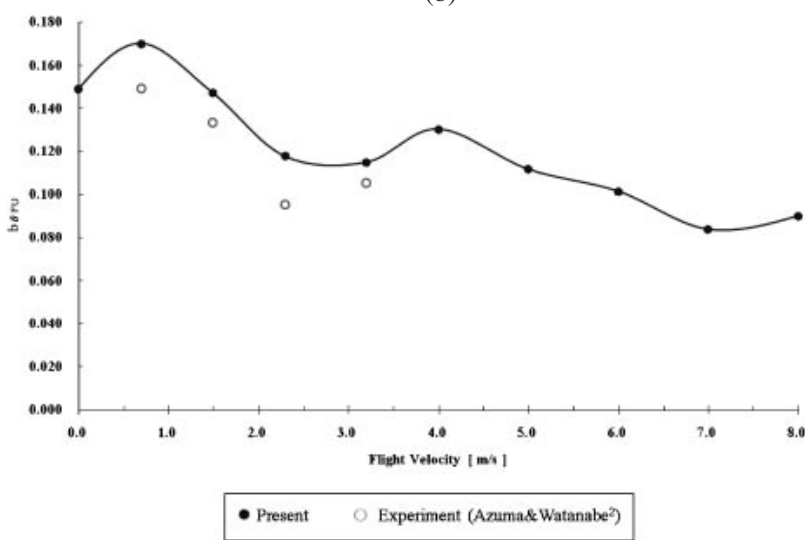

(c)
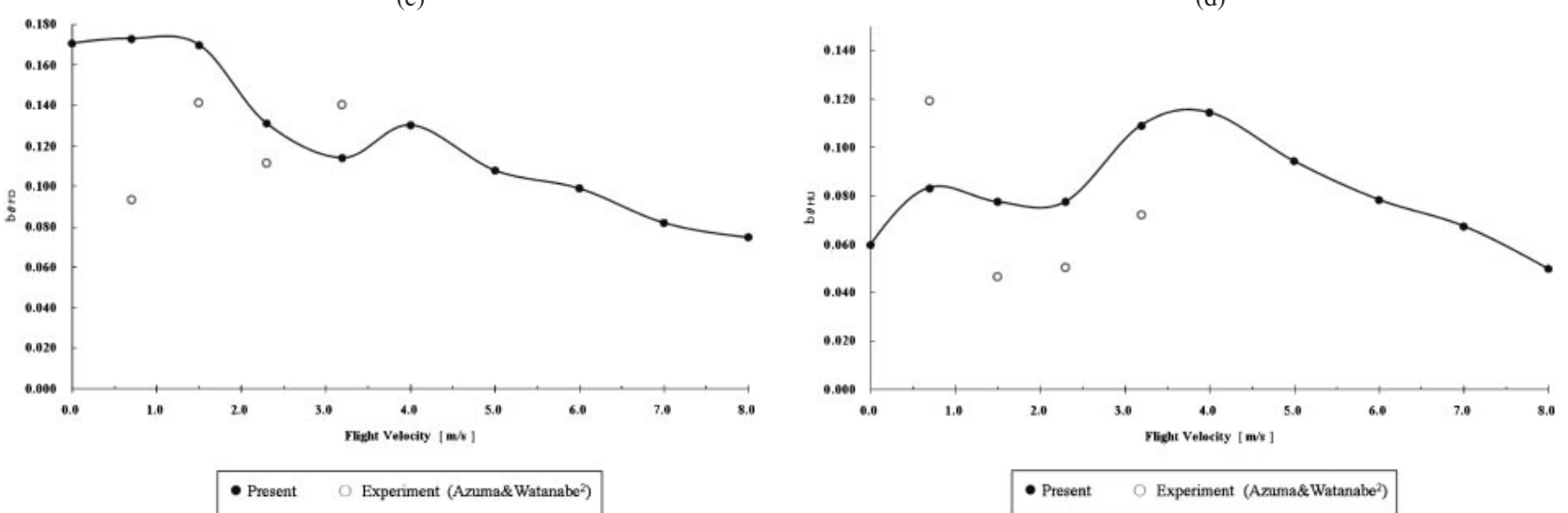

(e)

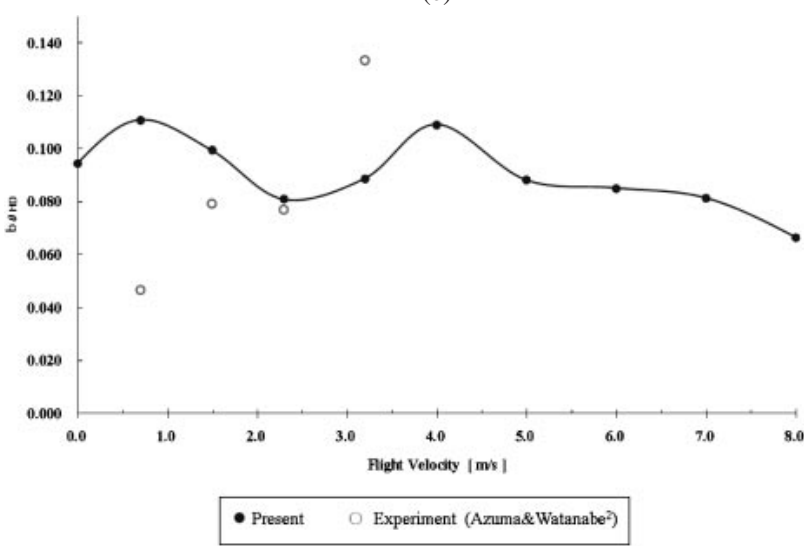

(f)

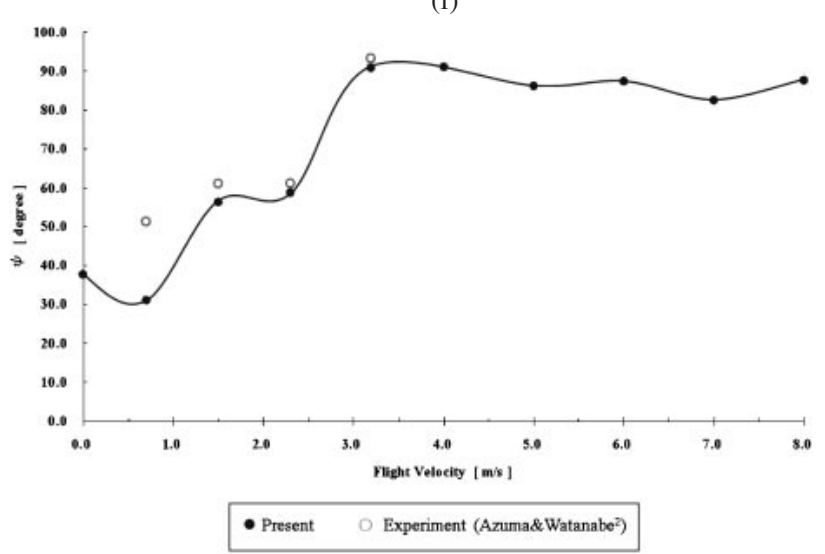

(h)

Fig. 12. (a) Variation of $\varphi_{\mathrm{S}}$ with respect to flight velocity. (b) Variation of $\varphi_{0 \mathrm{~F}}$ with respect to flight velocity. (c) Variation of $\varphi_{0 \mathrm{H}}$ with respect to flight velocity. (d) Variation of $b_{\theta \mathrm{FU}}$ with respect to flight velocity. (e) Variation of $b_{\theta \mathrm{FD}}$ with respect to flight velocity. (f) Variation of $b_{\theta \mathrm{HU}}$ with respect to flight velocity. (g) Variation of $b_{\theta \mathrm{HD}}$ with respect to flight velocity. (h) Variation of $\psi$ with respect to flight velocity. 

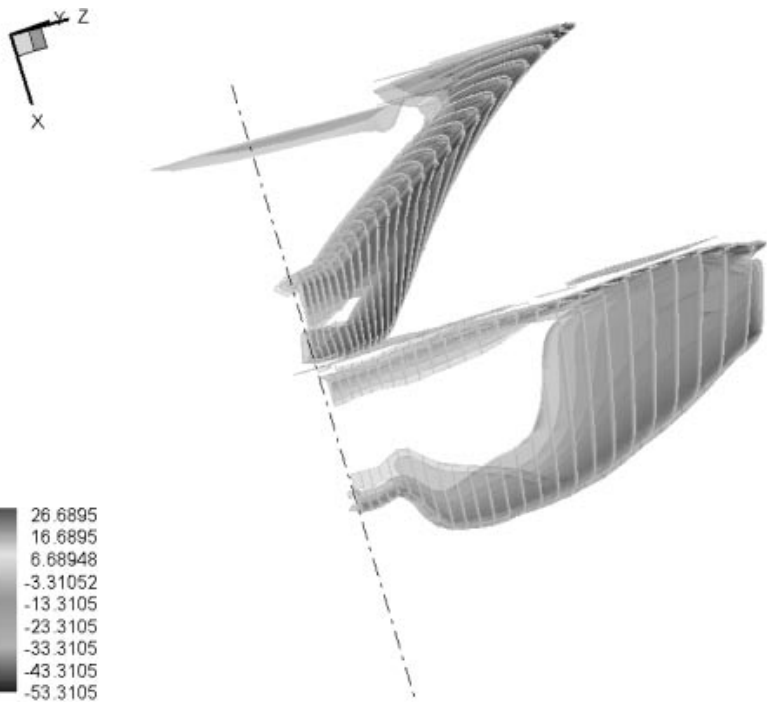

(a) $k t *=0$

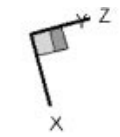

(b) $k t^{*}=\pi$

Fig. 13. Flow pattern (iso-vorticity) around fore- and hind-right-wings for $V_{\infty}=0 \mathrm{~m} / \mathrm{s} \quad$ (Reference velocity $V_{\mathrm{F}}=5.25 \mathrm{~m} / \mathrm{s}, \quad \varphi_{\mathrm{S}}=7.7^{\circ}$, $\psi=37.6^{\circ}$ ).

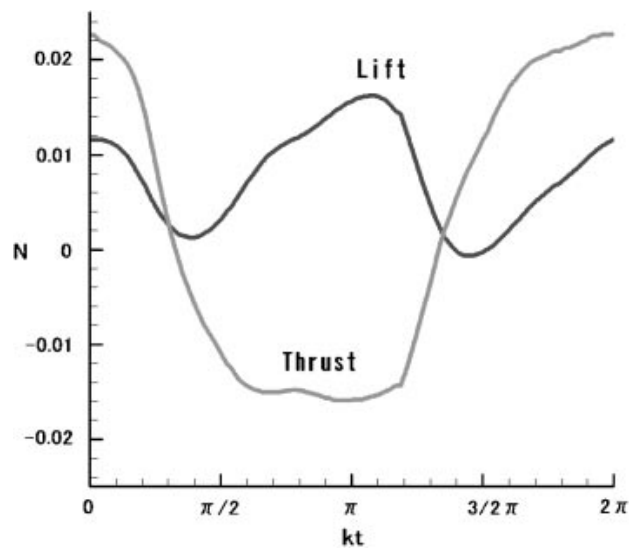

Fig. 14. Time histories of lift $L$ and thrust $T$ for $V_{\infty}=0 \mathrm{~m} / \mathrm{s}$.

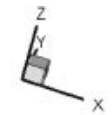

15.6227

$-20.3773$

$-443773$

-56.3773
-68.3773
-80.3773
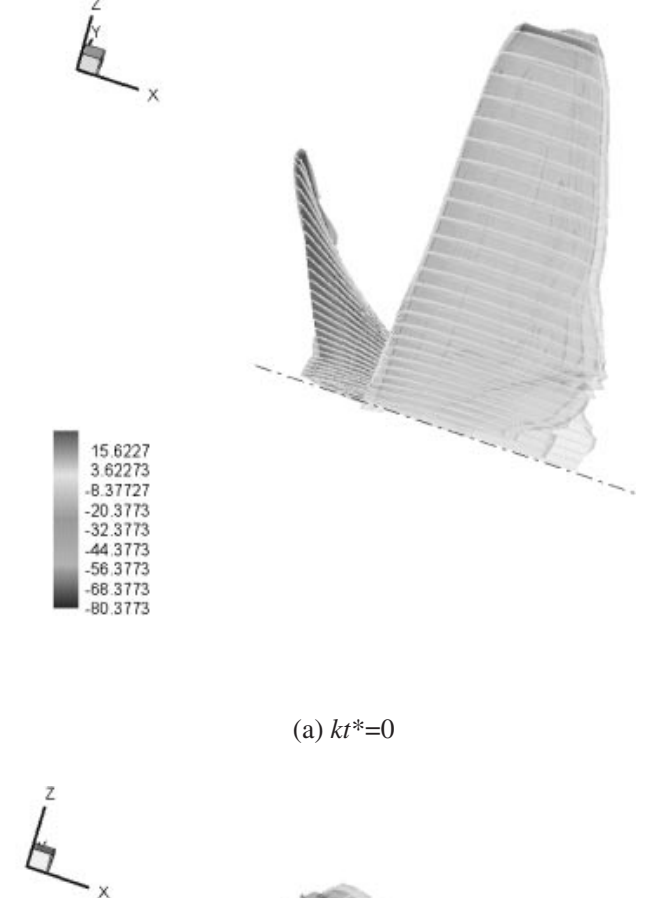

(b) $k t^{*}=\pi$

Fig. 15. Flow pattern (iso-vorticity) around fore- and hind-right-wings for $V_{\infty}=4.0 \mathrm{~m} / \mathrm{s}$ (Reference velocity $V_{\mathrm{F}}=4.16 \mathrm{~m} / \mathrm{s}, \varphi_{\mathrm{S}}=83.3^{\circ}$, $\left.\psi=91.0^{\circ}\right)$.

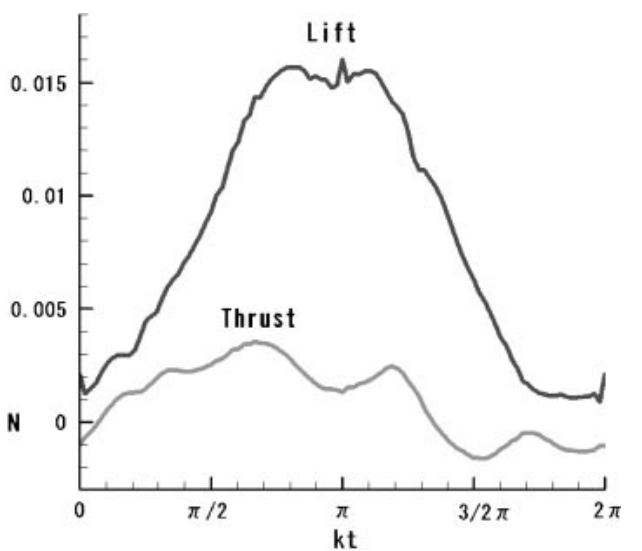

Fig. 16. Time histories of lift and thrust for $V_{\infty}=4.0 \mathrm{~m} / \mathrm{s}$. 


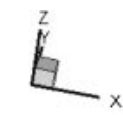

24.6607

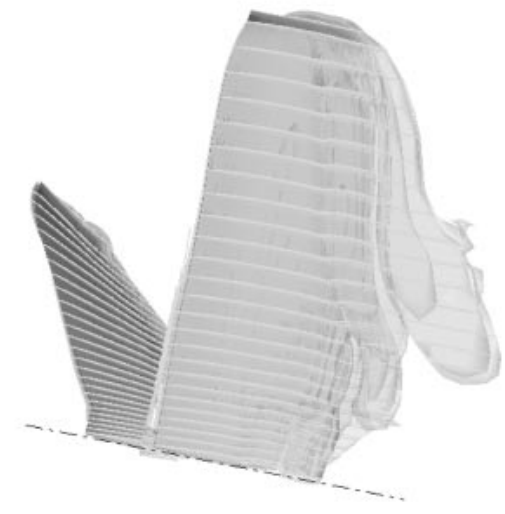

(a) $k t^{*}=0$
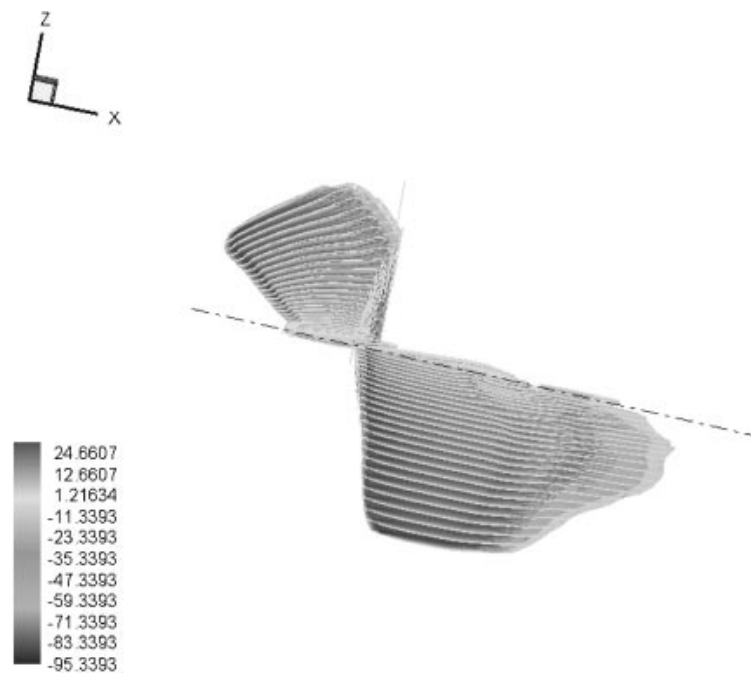

(b) $k t^{*}=\pi$

Fig. 17. Flow pattern (iso-vorticity) around fore- and hind-right-wings for $V_{\infty}=8.0 \mathrm{~m} / \mathrm{s}$ (Reference velocity $V_{\mathrm{F}}=4.58 \mathrm{~m} / \mathrm{s}, \varphi_{\mathrm{S}}=87.7^{\circ}$, $\psi=87.6^{\circ}$.

and $W$ are $0.00839 \mathrm{~N}(n=1.08), 0.00363 \mathrm{~N}\left(T^{*}=1.0\right)$ and $0.0452 W$, respectively.

\section{Conclusions}

We studied the optimum flapping wing motions of a dragonfly (A. p. julius), from hovering to cruising flight at various speeds, using a 3D Navier-Stokes code coupled with optimization algorithm. Using the optimization, we determined the minimum necessary power curve and the optimum flapping wing motions for various flight velocities, reaching the following conclusions. The minimum necessary power curve shows a typical U-shape; the necessary

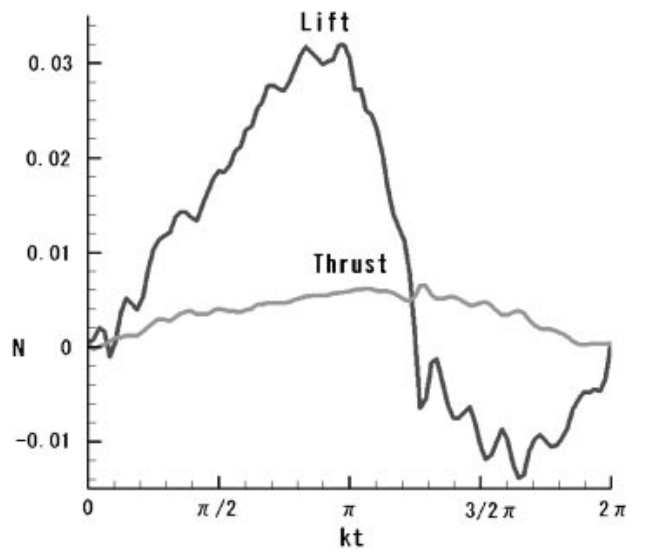

Fig. 18. Time histories of lift and thrust for $V_{\infty}=8.0 \mathrm{~m} / \mathrm{s}$.

power for hovering is $3.8 \times 10^{-2} \mathrm{~W}$, and the lowest necessary power is $1.1 \times 10^{-2} \mathrm{~W}$ at $V_{\infty}=4 \mathrm{~m} / \mathrm{s}$, which is about $1 / 3$ that of hovering. The maximum flight velocity is estimated more than $8 \mathrm{~m} / \mathrm{s}$. The stroke-plane angle for hovering is about $8^{\circ}$, and increases rapidly with increasing flight velocity, becoming $88^{\circ}$ at $V_{\infty}=8 \mathrm{~m} / \mathrm{s}$. The flapping amplitude of the forewing is about $30^{\circ}-60^{\circ}$, showing the maximum value for hovering, while that of the hindwing is around $20^{\circ}-40^{\circ}$. The rate of twist decreases with increasing flight velocity, and the phase advance angle of the flapping motion of the hindwing increases rapidly from about $30^{\circ}$ to $90^{\circ}$ with increasing flight velocity. The flow patterns around the fore- and hindwings at these optimum conditions were examined and show that large-scale flow separation around the wing is suppressed except at very low flight velocities, including hovering. This seems to be a main cause of efficient flight with minimum power consumption.

\section{References}

1) Weis-Fogh, T.: Quick-Estimate of Flight-Fitness in Hovering Animals, Including Novel Mechanism for Lift Production, J. Exp. Biol., 59 (1973), pp. 169-230.

2) Wakeling, J. M. and Ellington, C. P.: Dragonfly Flight II. Velocity, Accelerations and Kinematics of Flapping Flight, J. Exp. Biol., 200 (1997), pp. 557-582.

3) Azuma, A. and Watanabe, T.: Flight Performance of a Dragonfly, J. Exp. Biol., 137 (1988), pp. 221-252.

4) Isogai, K., Fujishiro, S., Saitoh, T., Yamamoto, M., Yamasaki, M. and Matsubara, M.: Unsteady Three-Dimensional Viscous Flow Simulation of a Dragonfly Hovering, AIAA J., 42 (2004), pp. 2053-2058.

5) Thomas, P. D. and Lombard, C. K.: Geometric Conservation Law and Its Application to Flow Computations on Moving Grids, AIAA J., 17 (1979), pp. 1030-1037.

6) Yamamoto, M. and Isogai, K.: Measurement of Unsteady Fluid Dynamic Forces for a Mechanical Dragonfly Model, AIAA J., 43 (2005), pp. 2475-2480.

7) Box, M. J.: A New Method of Constrained Optimization and a Comparison with Other Methods, Comput. J., 8 (1965), pp. 42-52. 